\title{
Prenatal exposure to antipsychotic medication does not increase odds of autism, ADHD
}

\section{BY PETER HESS}

20 AUGUST 2021

\section{Listen to this story: \\ https://www.spectrumnews.org/wp- content/uploads/2021/08/audio-227b29f6-da3b-4e21-9495-48c9ba89b395-encodings.mp3}

Children born to mothers who take antipsychotic medications during pregnancy do not have elevated odds of autism or attention deficit hyperactivity disorder (ADHD), nor are they more likely to be born preterm or underweight, according to a study released this past Monday in JAMA Internal Medicine.

Some women with schizophrenia, Tourette syndrome or bipolar disorder take antipsychotic drugs, such as aripiprazole, haloperidol or risperidone. Clinicians have long debated whether women should discontinue these medications during pregnancy out of concern for the drugs' effects on the developing fetus.

But children born to mothers who take antipsychotics during pregnancy and to those who do not take them have similar outcomes, the new work shows.

"Our findings do not support a recommendation for women to discontinue their regular antipsychotic treatment during pregnancy," says senior investigator Kenneth Man, research fellow at the University College London School of Pharmacy in the United Kingdom.

Prescribing antipsychotics during pregnancy can help prevent potentially dangerous psychotic episodes and ensure that an expectant mother can take care of herself, says Mady Hornig, associate professor of epidemiology at Columbia University, who was not involved in the study. "We certainly don't want to be cavalier about the use of any medication during pregnancy, but one 


\section{Spectrum | Autism Research News}

https://www.spectrumnews.org

also wants to balance out the implications of not treating."

Any apparent effects of antipsychotics on a developing fetus are likely due to the condition being treated, rather than the treatment, the study shows. New research on the link between prenatal antidepressant exposure and autism in children reaches a similar conclusion.

!function()\{"use strict";window.addEventListener("message",(function(e)\{if(void $0 !==$.data["datawrapper-height"])\{var t=document.querySelectorAll("iframe");for(var a in e.data["datawrapper-height"])for(var $r=0 ; r$ 